\title{
Stability of the Semi-Implicit Method for the Cahn-Hilliard Equation with Logarithmic Potentials
}

\author{
Dong $\mathrm{Li}^{1, *}$ and Tao Tang ${ }^{2}$ \\ ${ }^{1}$ Department of Mathematics, Hong Kong University of Science and \\ Technology, Clear Water Bay, Kowloon, Hong Kong \\ ${ }^{2}$ Division of Science and Technology, BNU-HKBU United International \\ College, Zhuhai 519087, Guangdong, China; and SUSTech International \\ Center for Mathematics, Southern University of Science and \\ Technology, Shenzhen 518055, Guangdong, China
}

Received 11 October 2020; Accepted (in revised version) 15 February 2021

\begin{abstract}
We consider the two-dimensional Cahn-Hilliard equation with logarithmic potentials and periodic boundary conditions. We employ the standard semi-implicit numerical scheme, which treats the linear fourth-order dissipation term implicitly and the nonlinear term explicitly. Under natural constraints on the time step we prove strict phase separation and energy stability of the semiimplicit scheme. This appears to be the first rigorous result for the semi-implicit discretization of the Cahn-Hilliard equation with singular potentials.
\end{abstract}

AMS subject classifications: 65M60, 35Q35

Key words: Cahn-Hilliard equation, logarithmic kernel, semi-implicit scheme, energy stability.

\section{Introduction}

Consider the 2D Cahn-Hilliard equation on $\Omega=\mathbb{T}^{2}=[-\pi, \pi)^{2}$ :

$$
\left\{\begin{array}{l}
\partial_{t} u=\Delta \mu=\Delta\left(-\nu \Delta u+F^{\prime}(u)\right), \quad(t, x) \in(0, \infty) \times \Omega, \\
\left.u\right|_{t=0}=u_{0}
\end{array}\right.
$$

\footnotetext{
${ }^{*}$ Corresponding author. Email addresses: madli@ust.hk (D. Li), tangt@sustech.edu.cn (T. Tang)
} 
where $u: \Omega \rightarrow(-1,1)$ is the order parameter of a two-phase system such as a binary alloy, and the term $\mu$ denotes the chemical potential. The two end-points $u= \pm 1$ correspond to pure states. The coefficient $\nu>0$ denotes mobility. In this paper we take it to be a constant parameter. The thermodynamic potential $F:(-1,1) \rightarrow \mathbb{R}$ is given by

$$
\begin{array}{ll}
F(u)=\frac{\theta}{2}((1+u) \ln (1+u)+(1-u) \ln (1-u))-\frac{\theta_{c}}{2} u^{2}, & 0<\theta<\theta_{c}, \\
f(u)=F^{\prime}(u)=-\theta_{c} u+\frac{\theta}{2} \ln \frac{1+u}{1-u}=:-\theta_{c} u+\tilde{f}(u), & F^{\prime \prime}(u)=\frac{\theta}{1-u^{2}}-\theta_{c},
\end{array}
$$

where the logarithmic part accounts for the entropy of mixing. The parameters $\theta$ and $\theta_{c}$ corresponds to the absolute temperature and the critical temperature respectively. Denote by $u_{+}>0$ the positive root of the equation $f(u)=0$ (see (1.2b)). Under the condition $0<\theta<\theta_{c}$ the potential $F$ takes the form of a double-well with two equal minima at $u_{+}$and $-u_{+}$which are usually called binodal points. One should note that the condition $0<\theta<\theta_{c}$ is of physical importance since it guarantees that that $F$ has a double-well form and phase separation can indeed occur. For $u_{s}=\left(1-\theta / \theta_{c}\right)^{\frac{1}{2}}$, the region $\left(-u_{s}, u_{s}\right)$, where $F^{\prime \prime}(u)<0$ is called the spinodal interval. If the quenching is shallow, i.e., the temperature $\theta$ is close to the absolute temperature $\theta_{c}$, then one can expand near $u=0$ and obtain the usual quartic polynomial approximation of the free energy.

The usual energy conservation takes the form:

$$
\frac{d}{d t} \mathcal{E}(u)=-\left\||\nabla|^{-1} \partial_{t} u\right\|_{2}^{2}, \quad \mathcal{E}(u)=\int_{\Omega}\left(\frac{1}{2} \nu|\nabla u|^{2}+F(u)\right) d x .
$$

Note that for $u \in(-1,1)$, the term $F(u)$ is bounded by an absolute constant, and the only coercive quantity in $\mathcal{E}(u)$ is the gradient term.

Remark 1.1. We note that the usual quartic polynomial approximation of the free energy $F(u)$ is given by (below the series converges for $u \in[-1,1]$ )

$$
\begin{aligned}
F(u) & =-\frac{\theta_{c}}{2} u^{2}+\theta \sum_{k=0}^{\infty} \frac{u^{2 k+2}}{(2 k+1)(2 k+2)} \\
& \approx F_{\text {quartic }}(u)=\frac{\theta}{2} \cdot \frac{u^{4}}{6}+\left(\frac{\theta}{2}-\frac{\theta_{c}}{2}\right) u^{2} .
\end{aligned}
$$

The standard double-well potential const $\cdot\left(u^{2}-1\right)^{2}$ corresponds to the specific choice $\theta / \theta_{c}=3 / 4$. However, this approximation introduces a nontrivial shift of the location of the minimum. Namely for the original free energy $F(u)$, its two equal minima 
occur at $\pm u_{+}$, where $u_{+}>0$ is the positive root of the equation $f(u)=0$ (see $(1.2 \mathrm{~b})$ ). In particular, $0<u_{+}<1$. In contrast, the standard double well potential $F_{\text {quartic,standard }}=$ $\left(u^{2}-1\right)^{2} / 4$ has minima at $u= \pm 1$. We should point out that, in view of the two minima $\pm u_{+}$, which are well inside the region $(-1,1)$ and singularity of the derivative of the potential, it is in some sense natural to expect strict phase separation for the evolution equation, i.e.,

$$
\|u\|_{\infty} \leq 1-\delta_{0}<1 \text { for some } \delta_{0}>0 .
$$

The strict phase separation turns out to play an important role in the rigorous analysis of (1.1).

Mathematically speaking, the system (1.1) can be recast as a gradient flow of a Ginzburg-Landau (GL) type energy functional $\psi(u)$ in $H^{-1}$, i.e.,

$$
\partial_{t} u=-\left.\frac{\delta \psi}{\delta u}\right|_{H^{-1}}=\Delta\left(\left.\frac{\delta \psi}{\delta u}\right|_{L^{2}}\right)
$$

where $\left.\frac{\delta \psi}{\delta u}\right|_{H^{-1}},\left.\frac{\delta \psi}{\delta u}\right|_{L^{2}}$ denote the standard variational derivatives in $H^{-1}$ and $L^{2}$ respectively, and

$$
\psi(u)=\int_{\Omega}\left(\frac{1}{2} \nu|\nabla u|^{2}+F(u)\right) d x .
$$

Here the gradient term in the GL energy accounts for surface tension effects, or more generally, short range interactions in the material. This particular form of energy functional can be derived from an approximation of a nonlocal term representing long range interactions [2]. In [10,11] Giacomin and Lebowitz considered a lattice gas model with certain long range Kac potentials, and gave a rigorous derivation of the nonlocal Cahn-Hilliard equation. Further results such as regularity and traveling waves on these and similar models can be found in $[12,13,16]$ and the references therein.

For the Cahn-Hilliard equation with constant mobility and logarithmic potentials, Elliott and Luckhaus in [7] considered the case of a multi-component mixture in a finite domain with Neumann boundary conditions and proved that if the initial data $u_{0} \in H^{1}$ satisfies $\left\|u_{0}\right\|_{\infty} \leq 1$ with space average in $(-1,1)$, then there exists a unique global solution $u \in C_{t}^{0} H^{-1} \cap L_{t}^{\infty} H_{x}^{1}, \partial_{t} u \in L_{t, \text { loc }}^{2} H_{x}^{-1}, \sqrt{t} \partial_{t} u \in L_{t, \text { loc }}^{2} H_{x}^{1}$ and $\|u\|_{\infty} \leq 1$. Furthermore, it was shown that the set $\{|u|=1\}$ has measure zero so that there are no singularities in the potential. The key idea in [7] is to use regularization and replace the logarithmic term by a smoothed version:

$$
\phi_{\epsilon}(r)= \begin{cases}\ln r, & r \geq \epsilon, \\ \ln \epsilon-1+\frac{r}{\epsilon}, & r<\epsilon .\end{cases}
$$


The main point is to derive $\epsilon$-independent estimates on the regularized problem and extract the desired solution in the vanishing $\epsilon$-limit. In [6], Debussche and Dettori adopted a different regularization of $F(u)$ :

$$
F_{N}(u)=-\frac{\theta_{c}}{2} u^{2}+\theta \sum_{k=0}^{N} \frac{u^{2 k+2}}{(2 k+1)(2 k+2)} .
$$

For $L^{2}$ or $H^{1}$ initial data $u_{0}$ with $\left\|u_{0}\right\|_{\infty} \leq 1, m\left(u_{0}\right) \in(-1,1)$ with either Neumann or periodic boundary conditions, they proved the existence and uniqueness of solutions as well as continuity of the semigroup. In [15], Miranwille and Zelik introduced another novel approximation by using the viscous Cahn-Hilliard equations, namely

$$
\left\{\begin{array}{l}
\epsilon \partial_{t} u+(-\Delta)_{N}^{-1} \partial_{t} u=\Delta u-f(u)+\langle f(u)\rangle, \quad \epsilon>0 \\
\left.\partial_{n} u\right|_{\partial \Omega}=0
\end{array}\right.
$$

where

$$
\langle v\rangle:=|\Omega|^{-1} \int_{\Omega} v(x) d x
$$

and $(-\Delta)_{N}^{-1}$ denotes the inverse Laplacian with Neumann boundary conditions acting on the space $L_{0}^{2}(\Omega)=\left\{v \in L^{2}(\Omega):\langle v\rangle=0\right\}$. In [1], Abels and Wilke employed a different approach based on the powerful theory of monotone operators. It is worthwhile pointing out that, to show the subgradient $\partial F(c)$ is single-valued (see Theorem 4.3 on [1, pp. 3183] and the proof therein), one needs some suitable approximation of the potential by smooth ones (since the derivative goes to $\pm \infty$ at the end-points) and carefully derive the limits. In a related work [14], Kenmochi, Niezgódka and Pawlow studied a very general version of Cahn-Hilliard equation involving a multivalued mapping by using sub-differential operator theory. The approach therein is based on several approximation procedures using smoothed equations and potentials. We note that more recently there has been some new developments on the analysis of the Cahn-Hilliard equation with singular potentials and dynamical boundary conditions $[17,18]$. Regarding the prior state of the art literature on these topics and more classical theory concerning long time behavior and attractors, we refer the interested readers to [3,17-19] and the references therein for more in-depth reviews and discussions.

There are some subtle technical difficulties associated with the numerical discretization of (1.1). We now point out two most pronounced issues. Denote $u^{n} \approx u\left(t_{n}\right)$ as the numerical solution at time step $t_{n}=n \tau$, where $\tau>0$ is the time step.

1. How do we guarantee that $u^{n} \in(-1,1)$ for all $n \geq 0$ ? 
2. How to ensure the energy decay property: $\mathcal{E}\left(u^{n+1}\right) \leq \mathcal{E}\left(u^{n}\right)$ for all $n \geq 0$ ?

One should note that the first issue is already present for the continuous PDE solutions. As was already mentioned earlier, the traditional route to solving this problem is via regularization of the nonlinearity or using the technique of sub-differential operators. The regularization technique can be transferred and modeled on the numerical discretization especially for the existence of solutions (for implicit schemes). Indeed in [8] developing upon the earlier work [7], Copetti and Elliott [8] considered a fully implicit Euler scheme applied to the Cahn-Hilliard equation with a finite element approximation in space. It was shown that under the condition that the time step $\tau$ is sufficiently small, and if the initial data satisfies $\left\|u_{0}\right\|_{\infty} \leq 1, \overline{u_{0}}<1-\delta<1$, then there exists a unique numerical solution for the implicit Euler discretization, satisfying $\left\|u^{n}\right\|_{\infty}<1$ for all $n \geq 1$. In [9] the authors generalized the approach in [8] to the multi-component Cahn-Hilliard flow. It should be noted that, due to the implicit treatment of the (concave) diffusion term, the energy stability and time step constraint is not unconditional in [8]. This can be rectified using D. Eyre's convex-splitting technique which is recently adopted in [5] using centered difference discretization in space. We note that the convex-splitting technique belongs to socalled partially implicit methods [20] for which the convex part of the nonlinearity is treated implicitly. By using a variational idea taking advantage of the singular nature of the nonlinearity, the numerical solution constructed in [5] can be guaranteed to lie in the interval $[-1,1]$ in each iteration. However, for semi-implicit methods, this line of argument completely breaks down, and to our best knowledge, this issue was completely open.

Whilst the first issue already presents itself a fundamental problem for semiimplicit methods, the second one is even more serious. As it turns out the explicit or implicit treatment of the nonlinear term can lead to a fundamental change of the energy stability of the associated iterative system. The analysis of energy stability gives a clear picture why implicit methods (or partially implicit methods) are usually favored/adopted in the literature (see also recent [21]). To elucidate the discussion we shall compare the usual semi-implicit methods with the implicit methods in the next two subsections. For simplicity we assume the ideal scenario that all $u^{n} \in(-1,1)$.

\section{The usual semi-implicit discretization case}

A typical semi-implicit discretization takes the form

$$
\frac{u^{n+1}-u^{n}}{\tau}=-\nu \Delta^{2} u^{n+1}+\Delta\left(f\left(u^{n}\right)\right)
$$


Multiplying both sides by $(-\Delta)^{-1}\left(u^{n+1}-u^{n}\right)$ (note that $\overline{u^{n+1}}=\overline{u^{n}}$, and see $(1.22)$ for the definition of $(-\Delta)^{-1}$ and $\bar{u}$ ) and integrating by parts, we obtain

$$
\frac{1}{\tau}\left\||\nabla|^{-1}\left(u^{n+1}-u^{n}\right)\right\|_{2}^{2}+\frac{\nu}{2}\left\|\nabla\left(u^{n+1}-u^{n}\right)\right\|_{2}^{2}+\mathcal{E}\left(u^{n+1}\right)-\mathcal{E}\left(u^{n}\right)=\int_{\Omega} H_{1} d x,
$$

where $|\nabla|^{-1}=(-\Delta)^{-\frac{1}{2}}($ see $(1.21))$, and $H_{1}=F\left(u^{n+1}\right)-F\left(u^{n}\right)-f\left(u^{n}\right)\left(u^{n+1}-u^{n}\right)$.

Now recall that

$$
F^{\prime \prime}(\xi)=\frac{\theta}{1-\xi^{2}}-\theta_{c}
$$

Clearly then

$$
H_{1}=\frac{1}{2}\left(\frac{\theta}{1-\xi_{0}^{2}}-\theta_{c}\right) \cdot\left(u^{n+1}-u^{n}\right)^{2},
$$

where $\xi_{0}$ is a function with values sandwiched between $u^{n}$ and $u^{n+1}$. Note that on the LHS of (1.10), we have the usual estimate

$$
\frac{1}{\tau}\left\||\nabla|^{-1}\left(u^{n+1}-u^{n}\right)\right\|_{2}^{2}+\frac{\nu}{2}\left\|\nabla\left(u^{n+1}-u^{n}\right)\right\|_{2}^{2} \geq \sqrt{\frac{2 \nu}{\tau}}\left\|u^{n+1}-u^{n}\right\|_{2}^{2} .
$$

However even for very small $\tau>0$, this is in-sufficient to control the singular pre-factor $\frac{1}{1-\xi_{0}^{2}}$ in the $H_{1}$-term which could potentially become rather large when $\xi_{0} \rightarrow \pm 1$.

\section{The usual implicit discretization case}

A typical implicit discretization takes the form

$$
\frac{u^{n+1}-u^{n}}{\tau}=-\nu \Delta^{2} u^{n+1}+\Delta\left(f\left(u^{n+1}\right)\right)
$$

Multiplying both sides by $(-\Delta)^{-1}\left(u^{n+1}-u^{n}\right)$ and integrating, we obtain

$$
\frac{1}{\tau}\left\||\nabla|^{-1}\left(u^{n+1}-u^{n}\right)\right\|_{2}^{2}+\frac{\nu}{2}\left\|\nabla\left(u^{n+1}-u^{n}\right)\right\|_{2}^{2}+\mathcal{E}\left(u^{n+1}\right)-\mathcal{E}\left(u^{n}\right)=\int_{\Omega} H_{2} d x,
$$

where $H_{2}=F\left(u^{n+1}\right)-F\left(u^{n}\right)-f\left(u^{n+1}\right)\left(u^{n+1}-u^{n}\right)$.

Now recall again that $F^{\prime \prime}(\xi)=\frac{\theta}{1-\xi^{2}}-\theta_{c}$. Clearly then

$$
\begin{aligned}
H_{2} & =-F\left(u^{n}\right)+F\left(u^{n+1}\right)+f\left(u^{n+1}\right)\left(u^{n}-u^{n+1}\right) \\
& =\frac{1}{2}\left(-\frac{\theta}{1-\xi_{0}^{2}}+\theta_{c}\right) \cdot\left(u^{n}-u^{n+1}\right)^{2},
\end{aligned}
$$


where $\xi_{0}$ is a function with values sandwiched between $u^{n}$ and $u^{n+1}$. Note that on the LHS of (1.14), we have the usual estimate

$$
\begin{aligned}
& \frac{1}{\tau}\left\||\nabla|^{-1}\left(u^{n+1}-u^{n}\right)\right\|_{2}^{2}+\frac{\nu}{2}\left\|\nabla\left(u^{n+1}-u^{n}\right)\right\|_{2}^{2} \\
\geq & \sqrt{\frac{2 \nu}{\tau}}\left\|u^{n+1}-u^{n}\right\|_{2}^{2} \geq \frac{1}{2} \theta_{c}\left\|u^{n+1}-u^{n}\right\|_{2}^{2},
\end{aligned}
$$

if $0<\tau \leq 8 \nu / \theta_{c}^{2}$. On the other hand, note that the singular term $-\frac{1}{1-\xi_{0}^{2}}$ is always negative (provided we guarantee that $u^{n}$ and $u^{n+1}$ always stay inside the interval $(-1,1))$. Thus the energy decay property can be expected for implicit methods.

Now, from the above comparative discussion in the preceding two subsections, it is clear that there are nontrivial technical obstacles for the semi-implicit methods applied on the Cahn-Hilliard equation with logarithmic potentials. Nevertheless, the purpose of this work is to introduce a new framework to settle these open issues.

Consider the following semi-implicit discretization of (1.1):

$$
\left\{\begin{array}{l}
\frac{u^{n+1}-u^{n}}{\tau}=-\nu \Delta^{2} u^{n+1}-\theta_{c} \Delta u^{n+1}+\Delta\left(\tilde{f}\left(u^{n}\right)\right), \quad n \geq 0 \\
u^{0}=u_{0}
\end{array}\right.
$$

where $\tilde{f}(u)=\frac{\theta}{2} \ln \left(\frac{1+u}{1-u}\right)$. The relation of $\tilde{f}(u)$ with $f(u)$ is given by (1.2b).

Theorem 1.1 (Stability of the semi-implicit discretization scheme). Consider the implicit-explicit scheme (1.17) for the phase field equation (1.1) with the logarithmic potential (1.2a). Assume the initial data $u_{0} \in H^{5}\left(\mathbb{T}^{2}\right)$ and $\left\|u_{0}\right\|_{\infty} \leq 1-\delta_{0}$ for some $\delta_{0} \in(0,1)$. There exists $\tau_{0}=\tau_{0}\left(\left\|u_{0}\right\|_{H^{5}}, \delta_{0}, \nu, \theta, \theta_{c}\right)>0$ such that for any $0<\tau \leq \tau_{0}$, the following hold for (1.17):

1. Unique solvability and propagation of Sobolev regularity. The iterates $u^{n}$ are well-defined for all $n \geq 1$. Furthermore, $\sup _{n \geq 1}\left\|u^{n}\right\|_{H^{5}\left(\mathbb{T}^{2}\right)} \leq A_{1}<\infty$ for some $A_{1}$ depending only on $\left(\left\|u_{0}\right\|_{H^{5}}, \delta_{0}, \nu, \theta, \theta_{c}\right)$.

2. Strict phase separation. There exists a constant $\delta_{1} \in(0,1)$ depending only on $\left(\left\|u_{0}\right\|_{H^{5}}, \delta_{0}, \nu, \theta, \theta_{c}\right)$, such that $\sup _{n \geq 1}\left\|u^{n}\right\|_{\infty} \leq 1-\delta_{1}$.

3. Energy stability. $\mathcal{E}\left(u^{n+1}\right) \leq \mathcal{E}\left(u^{n}\right)$ for all $n \geq 0$.

To prove Theorem 1.1, we introduce a new strategy which concurrently establishes the strict phase separation and uniform Sobolev regularity of the iterates $u^{n}$ through an inductive procedure. Besides using the discrete energy inequality to 
control $H^{1}$-norm of $u^{n}$, we employ several bootstrapping long time estimates on the discrete chemical potential

$$
K^{n}=-\nu \Delta u^{n}-\theta_{c} u^{n}+\tilde{f}\left(u^{n}\right)
$$

to gain uniform-in-time higher Sobolev bounds. This part of the argument is technical and we have to appeal to a delicate dichotomy argument to eliminate some sporadic drift of higher norms of $K^{n}$ (see Subsection 2.3 for more details). The strict phase separation property of $u^{n}$ can be deduced through a uniform estimate on the quantity

$$
g^{n}=\tilde{f}\left(u^{n}\right)=\frac{\theta}{2} \ln \left(\frac{1+u^{n}}{1-u^{n}}\right)
$$

which in turn is obtained by analyzing a nonlinear elliptic problem connecting $g^{n}$ to $K^{n}$. A subtle point in the whole analysis is to obtain uniform in time estimates which are largely independent of the induction hypothesis. In order not to overburden the reader with notations and keep the analysis relatively simple, we do not optimize the regularity assumption on initial data, and we do not spell out the precise dependence of the time step constraint on various parameters. All these issues and further generalizations will be addressed in forthcoming works.

Remark 1.2. We stress again that the assumption $\left\|u_{0}\right\|_{\infty} \leq 1-\delta_{0}$ is quite natural from the point of view that the free energy has two equal minima well inside the interval $(-1,1)$ and its derivative blows up as $u \rightarrow \pm 1$.

Remark 1.3. A variant of the scheme (1.17) is:

$$
\frac{u^{n+1}-u^{n}}{\tau}=-\nu \Delta^{2} u^{n+1}+\Delta\left(f\left(u^{n}\right)\right)
$$

where

$$
f(u)=-\theta_{c} u+\frac{\theta}{2} \ln \left(\frac{1+u}{1-u}\right) .
$$

Theorem 1.1 also holds for this case. Compared with (1.17), a slight difference is the solvability of $u^{n+1}$ in the numerical scheme. In the former case the time step has to be taken suitably small so that $u^{n+1}$ can be uniquely solved from $u^{n}$. In the latter case (i.e., (1.18)) the solvability is not an issue and one can uniquely solve $u^{n+1}$ for any $\tau>0$.

Remark 1.4. From a more practical point view, one should consider the spectral Galerkin truncated system:

$$
\left\{\begin{array}{l}
\frac{u^{n+1}-u^{n}}{\tau}=-\nu \Delta^{2} u^{n+1}-\theta_{c} \Delta u^{n+1}+\Delta \Pi_{N}\left(\tilde{f}\left(u^{n}\right)\right), \quad n \geq 0, \\
u^{0}=\Pi_{N} u_{0}
\end{array}\right.
$$


where $\Pi_{N}$ is the projection into first $N$ Fourier modes. With minor modifications our analysis can be extended to this case. Note that in this case for the phase separation property to hold, we need to impose it on $u^{0}=\Pi_{N} u_{0}$ since $\Pi_{N}$ is not a continuous operator in $L^{\infty}$. Alternatively by using the high regularity of $u_{0}$, one can show that

$$
\lim _{N \rightarrow \infty}\left\|u^{0}-\Pi_{N} u_{0}\right\|_{\infty}=\lim _{N \rightarrow \infty}\left\|\Pi_{>N} u_{0}\right\|_{\infty}=0
$$

As an immediate application of Theorem 1.1 (and to make this paper selfcontained), we obtain the following wellposedness result for the continuous PDE solution to (1.1). As a matter of fact this approach can be refined to yield a new wellposedness and regularity theory for the continuous case which we will address elsewhere. For simplicity we do not lower the regularity assumption on the initial data.

Corollary 1.1 (Existence and uniqueness of the PDE solution). Assume the initial data $u_{0} \in H^{s}\left(\mathbb{T}^{2}\right), s \geq 5$ and $\left\|u_{0}\right\|_{\infty} \leq 1-\delta_{0}$ for some $\delta_{0} \in(0,1)$. Then corresponding to $u_{0}$ there exists a unique global solution $u \in C_{t}^{0} H_{x}^{s} \cap C_{t}^{1} H_{x}^{s-4}$ to (1.1) satisfying

$$
\sup _{0 \leq t<\infty}\|u(t)\|_{H^{s}}<\infty \quad \text { and } \quad \sup _{0 \leq t<\infty}\|u(t)\|_{\infty} \leq 1-\delta_{1}
$$

for some $\delta_{1} \in(0,1)$.

Our final result is the error analysis for the semi-implicit scheme. A similar result also holds for the variant (1.18).

Theorem 1.2 (Error analysis). Let $\nu>0$. Assume the initial data $u_{0} \in H^{5}\left(\mathbb{T}^{2}\right)$ and $\left\|u_{0}\right\|_{\infty} \leq 1-\delta_{0}$ for some $\delta_{0} \in(0,1)$. Let $u^{n}$ be the corresponding numerical solution constructed in Theorem 1.1. Let $u(t)$ the exact PDE solution to (1.1) constructed in Corollary 1.1. Let

$$
0<\tau \leq \min \left\{\tau_{0}, \frac{\nu}{4 \theta_{c}^{2}}\right\}
$$

where $\tau_{0}$ is the same as in Theorem 1.1. Define $t_{m}=m \tau, m \geq 1$. Then

$$
\left\|u\left(t_{m}\right)-u^{m}\right\|_{2} \leq C_{1} e^{C_{2} t_{m}} \tau .
$$

Here, $C_{1}, C_{2}>0$ depends on $\left(u_{0}, \delta_{0}, \nu, \theta_{c}, \theta\right)$.

The rest of this paper is organized as follows. In Section 2 we give the proof of Theorem 1.1. Section 3 is devoted to the proof of Corollary 1.1. In Section 4 we complete the error analysis and give the proof of Theorem 1.2. In Section 5 we give some concluding remarks. 
Notation 1.1. For any real number $a \in \mathbb{R}$, we denote by $a+$ the quantity $a+\epsilon$ for sufficiently small $\epsilon>0$. The numerical value of $\epsilon$ is unimportant, and the needed smallness of $\epsilon$ is usually clear from the context. The notation $a-$ is similarly defined. This notation is particularly handy for interpolation inequalities. For example we shall use the notation

$$
\|f\|_{\infty-}
$$

to denote $\|f\|_{p}$ for all large $p<\infty$.

For any two quantities $X$ and $Y$, we denote $X \lesssim Y$ or $X=\mathcal{O}(Y)$ if $X \leq C Y$ for some constant $C>0$. Similarly $X \gtrsim Y$ if $X \geq C Y$ for some $C>0$. We denote $X \sim Y$ if $X \lesssim Y$ and $Y \lesssim X$. The dependence of the constant $C$ on other parameters or constants is usually clear from the context and we will often suppress this dependence. We denote $X \lesssim Z_{1}, \cdots, Z_{m} Y$ if $X \leq C Y$, where the constant $C$ depends on the parameters $Z_{1}, \cdots, Z_{m}$. For any quantities $X_{1}, X_{2}, \cdots, X_{N}$, we denote by $C\left(X_{1}, \cdots, X_{N}\right)$ or $C_{X_{1}, \cdots, X_{N}}$ a positive constant depending on $\left(X_{1}, \cdots, X_{N}\right)$.

We denote by $\mathbb{T}^{2}=[-\pi, \pi)^{2}$ the usual periodic torus in two dimensions. For a function $f: \mathbb{T}^{2} \rightarrow \mathbb{R}$, we denote by

$$
\bar{f}=\frac{1}{(2 \pi)^{2}} \int_{\mathbb{T}^{2}} f(x) d x
$$

the average/mean value of $f$ on $\mathbb{T}^{2}$. We adopt the following convention for the usual Fourier transform on $\mathbb{T}^{2}$ (below assume $f \in C^{\infty}$ for simplicity):

$$
\begin{aligned}
& (\mathcal{F} f)(k)=\widehat{f}(k)=(2 \pi)^{-2} \int_{\mathbb{T}^{2}} f(x) e^{-i x \cdot k} d x, \quad k \in \mathbb{Z}^{2}, \\
& f(x)=\sum_{k \in \mathbb{Z}^{2}} \widehat{f}(k) e^{i k \cdot x} .
\end{aligned}
$$

We denote by $|\nabla|^{s}=(-\Delta)^{\frac{s}{2}}$ the operator corresponding to the symbol $|k|^{s}$ such that

$$
\widehat{|\nabla|^{s} f}(k)=|k|^{s} \widehat{f}(k) \text {. }
$$

Note that for $s<0,|\nabla|^{s} f$ is only defined for smooth functions $f$ with $\widehat{f}(0)=0$. For example, if $f \in L^{1}\left(\mathbb{T}^{2}\right)$ and $\bar{f}=0$ (thus $\widehat{f}(0)=0$ ), we can define

$$
\mathcal{F}\left((-\Delta)^{-1} f\right)(k)=\frac{1}{|k|^{2}} \widehat{f}(k), \quad \forall 0 \neq k \in \mathbb{Z}^{2} .
$$

In yet other words, $(-\Delta)^{-1}$ corresponds to the Fourier multiplier $1 /|k|^{2}$ acting on $L^{1}$ functions whose zero ${ }^{\text {th }}$ mode is zero.

For $f, g \in L^{2}\left(\mathbb{T}^{2} \rightarrow \mathbb{R}\right)$, we denote by $\langle$,$\rangle the usual L^{2}$-pairing:

$$
\langle f, g\rangle=\int_{\mathbb{T}^{2}} f(x) g(x) d x .
$$




\section{Proof of Theorem 1.1}

For simplicity we assume $\nu=1$ in (1.1). Let us consider the following semi-implicit scheme:

$$
\frac{u^{n+1}-u^{n}}{\tau}=-\Delta^{2} u^{n+1}-\theta_{c} \Delta u^{n+1}+\Delta\left(\tilde{f}\left(u^{n}\right)\right),
$$

where

$$
\tilde{f}(u)=\frac{\theta}{2} \ln \left(\frac{1+u}{1-u}\right) .
$$

Then

$$
u^{n+1}=\frac{1}{1+\tau \Delta^{2}+\tau \theta_{c} \Delta} u^{n}+\frac{\tau \Delta}{1+\tau \Delta^{2}+\tau \theta_{c} \Delta}\left(\tilde{f}\left(u^{n}\right)\right) .
$$

Note that for $0 \neq k \in \mathbb{Z}^{2}$,

$$
1+\tau|k|^{4} \geq 2 \sqrt{\tau}|k|^{2} \geq \theta_{c} \tau|k|^{2}
$$

if $0<\tau \leq \frac{4}{\theta_{c}^{2}}$. We shall assume the slightly stronger condition $0<\tau \leq \frac{2}{\theta_{c}^{2}}$ to ensure solvability.

For convenience we shall sometimes denote

$$
g^{n}=\tilde{f}\left(u^{n}\right)=\frac{\theta}{2} \ln \frac{1+u^{n}}{1-u^{n}} .
$$

The inductive assumption is:

$$
\left\|g^{n}\right\|_{H^{2}} \leq A_{0}<\infty, \quad\left\|u^{n}\right\|_{H^{5}} \leq A_{1}<\infty .
$$

The choice of the constants $A_{0}$ and $A_{1}$ will become clear in the course of the proof. The base step $n=0$ clearly holds true. In the rest of the proof we shall focus on the induction step $n \Rightarrow n+1$ for general $n$.

From the estimate of $g^{n}$, it follows that $\left\|u^{n}\right\|_{\infty} \leq 1-\delta_{1}<1$ for some $\delta_{1}>0$. Also clearly by using the iterative relation,

$$
\left\|u^{n+1}\right\|_{H^{5}} \leq C_{A_{1}}<\infty
$$

Thus

$$
\left\|P_{>N}\left(u^{n+1}-u^{n}\right)\right\|_{\infty} \leq \frac{\delta_{1}}{4}
$$


if $N$ is sufficiently large (here $P_{>N}$ is the usual Littlewood-Paley projector adapted to frequency $|k| \gtrsim N)$. Now

$$
\begin{aligned}
& \left\|P_{\leq N}\left(u^{n+1}-u^{n}\right)\right\|_{\infty} \\
\leq & \left\|P_{\leq N} \frac{\Delta^{2}+\theta_{c} \Delta}{1+\tau \Delta^{2}+\tau \theta_{c} \Delta} u^{n}\right\|_{\infty}+\tau\left\|P_{\leq N} \frac{\Delta}{1+\tau \Delta^{2}+\tau \theta_{c} \Delta}\left(\tilde{f}\left(u^{n}\right)\right)\right\|_{\infty} \\
\leq & \mathcal{O}(\tau) \leq \frac{\delta_{1}}{4},
\end{aligned}
$$

if $\tau>0$ is sufficiently small. It follows that we can guarantee $\left\|u^{n+1}\right\|_{\infty} \leq 1-\delta_{1} / 2$.

We now divide the rest of the proof into several steps. The following notation will be used.

Notation 2.1. Throughout the rest of this proof, we shall denote by $C$ a generic constant depending only $\left(\left\|u_{0}\right\|_{H^{5}}, \delta_{0}, \theta, \theta_{c}\right)$. The value of $C$ can change from line to line. Sometimes for a quantity $X$ we use the notation $X \lesssim 1$ to denote $X \leq C$. We denote by $C_{A_{1}}$ a constant whose value depends on $A_{1}$. The value of $C_{A_{1}}$ may vary from line to line.

\subsection{Discrete energy estimate of $u^{n+1}$}

Multiplying both sides of $(2.1)$ by $(-\Delta)^{-1}\left(u^{n+1}-u^{n}\right)$ and integrating (Taylor expand $\tilde{F}\left(u^{n+1}\right)$ around $\left.\tilde{F}\left(u^{n}\right)\right)$, we obtain

$$
\begin{aligned}
& \frac{1}{\tau}\left\||\nabla|^{-1}\left(u^{n+1}-u^{n}\right)\right\|_{2}^{2}+\frac{1}{2}\left\|\nabla\left(u^{n+1}-u^{n}\right)\right\|_{2}^{2}+\mathcal{E}\left(u^{n+1}\right)-\mathcal{E}\left(u^{n}\right) \\
= & \frac{\theta_{c}}{2}\left\|u^{n+1}-u^{n}\right\|_{2}^{2}+\frac{1}{2} \int_{\Omega} \frac{\theta}{1-\xi_{n+1}^{2}}\left(u^{n+1}-u^{n}\right)^{2} d x,
\end{aligned}
$$

where $\xi_{n+1}$ is between $u^{n}$ and $u^{n+1}$. Since

$$
\left\|u^{n}\right\|_{\infty} \leq 1-\delta_{1} \quad \text { and } \quad\left\|u^{n+1}\right\|_{\infty} \leq 1-\frac{\delta_{1}}{2}
$$

we obtain

$$
\left\|\xi_{n+1}\right\|_{\infty} \leq 1-\frac{\delta_{1}}{2}
$$

Now note that

$$
\begin{aligned}
& \frac{1}{\tau}\left\||\nabla|^{-1}\left(u^{n+1}-u^{n}\right)\right\|_{2}^{2}+\frac{1}{2}\left\|\nabla\left(u^{n+1}-u^{n}\right)\right\|_{2}^{2} \\
\geq & 2 \sqrt{\frac{1}{8 \tau}}\left\|u^{n+1}-u^{n}\right\|_{2}^{2}+\frac{1}{2 \tau}\left\||\nabla|^{-1}\left(u^{n+1}-u^{n}\right)\right\|_{2}^{2}+\frac{1}{4}\left\|\nabla\left(u^{n+1}-u^{n}\right)\right\|_{2}^{2} .
\end{aligned}
$$


Thus if $\tau>0$ is sufficiently small such that

$$
2 \sqrt{\frac{1}{8 \tau}} \geq \frac{\theta_{c}}{2}+\frac{1}{2} \frac{\theta}{1-\left(1-\frac{\delta_{1}}{2}\right)^{2}},
$$

we can guarantee the energy stability:

$$
\frac{1}{2 \tau}\left\||\nabla|^{-1}\left(u^{n+1}-u^{n}\right)\right\|_{2}^{2}+\frac{1}{4}\left\|\nabla\left(u^{n+1}-u^{n}\right)\right\|_{2}^{2}+\mathcal{E}\left(u^{n+1}\right)-\mathcal{E}\left(u^{n}\right) \leq 0 .
$$

This also yields

$$
\frac{1}{2 \tau} \sum_{j=0}^{n}\left\||\nabla|^{-1}\left(u^{j+1}-u^{j}\right)\right\|_{2}^{2}+\frac{1}{4} \sum_{j=0}^{n}\left\|\nabla\left(u^{j+1}-u^{j}\right)\right\|_{2}^{2} \leq \mathcal{E}\left(u^{0}\right) .
$$

\subsection{Preliminary estimate of $K^{n+1}$}

Denote

$$
K^{n+1}=-\Delta u^{n+1}-\theta_{c} u^{n+1}+\tilde{f}\left(u^{n+1}\right) .
$$

Note that

$$
\frac{u^{n+1}-u^{n}}{\tau}=\Delta K^{n+1}+\Delta\left(\tilde{f}\left(u^{n}\right)-\tilde{f}\left(u^{n+1}\right)\right) .
$$

Lemma 2.1. It holds that

$$
\left\|\nabla\left(\tilde{f}\left(u^{n+1}\right)-\tilde{f}\left(u^{n}\right)\right)\right\|_{2} \leq C_{A_{1}}\left\|\nabla\left(u^{n+1}-u^{n}\right)\right\|_{2} .
$$

Thus if $\tau C_{A_{1}}^{2} \leq 1$, we have

$$
\tau\left\|\nabla\left(\tilde{f}\left(u^{n+1}\right)-\tilde{f}\left(u^{n}\right)\right)\right\|_{2}^{2} \leq \frac{1}{2}\left\|\nabla\left(u^{n+1}-u^{n}\right)\right\|_{2}^{2} .
$$

Proof. We write

$$
\tilde{f}\left(u^{n+1}\right)-\tilde{f}\left(u^{n}\right)=\alpha_{n+1} \cdot\left(u^{n+1}-u^{n}\right),
$$

where

$$
\alpha_{n+1}=\int_{0}^{1} \tilde{f}^{\prime}\left(u^{n}+\theta\left(u^{n+1}-u^{n}\right)\right) d \theta
$$

Since

$$
\left\|u^{n}\right\|_{\infty} \leq 1-\delta_{1}, \quad\left\|u^{n+1}\right\|_{\infty} \leq 1-\frac{\delta_{1}}{2} \quad \text { and } \quad\left\|u^{n+1}\right\|_{H^{5}} \leq C_{A_{1}}
$$


we clearly have

$$
\begin{aligned}
& \left\|\nabla\left(\tilde{f}\left(u^{n+1}\right)-\tilde{f}\left(u^{n}\right)\right)\right\|_{2} \\
\leq & \left\|\nabla \alpha_{n+1}\right\|_{\infty}\left\|u^{n+1}-u^{n}\right\|_{2}+\left\|\alpha_{n+1}\right\|_{\infty}\left\|\nabla\left(u^{n+1}-u^{n}\right)\right\|_{2} \\
\leq & C_{A_{1}}\left\|\nabla\left(u^{n+1}-u^{n}\right)\right\|_{2},
\end{aligned}
$$

where we have used the Poincaré inequality

$$
\left\|\nabla\left(u^{n+1}-u^{n}\right)\right\|_{2} \geq\left\|u^{n+1}-u^{n}\right\|_{2} .
$$

Thus, we complete the proof.

By Lemma 2.1, (2.8) and (2.7), it follows that for sufficiently small $\tau$, we have

$$
\begin{aligned}
& \tau \sum_{j=0}^{n}\left\|\nabla K^{j+1}\right\|_{2}^{2} \\
\leq & 2 \sum_{j=0}^{n} \frac{\left\||\nabla|^{-1}\left(u^{j+1}-u^{j}\right)\right\|_{2}^{2}}{\tau}+2 \sum_{j=0}^{n} \tau\left\|\nabla\left(\tilde{f}\left(u^{j+1}\right)-\tilde{f}\left(u^{j}\right)\right)\right\|_{2}^{2} \leq C .
\end{aligned}
$$

\subsection{Long time estimate of $K^{n+1}$}

Now we consider the evolution equation for $K^{n+1}$. We have

$$
\begin{aligned}
\frac{K^{n+1}-K^{n}}{\tau}=- & \Delta^{2} K^{n+1}-\theta_{c} \Delta K^{n+1}+\frac{\tilde{f}\left(u^{n+1}\right)-\tilde{f}\left(u^{n}\right)}{\tau} \\
& +\Delta^{2}\left(\tilde{f}\left(u^{n+1}\right)-\tilde{f}\left(u^{n}\right)\right)+\theta_{c} \Delta\left(\tilde{f}\left(u^{n+1}\right)-\tilde{f}\left(u^{n}\right)\right) .
\end{aligned}
$$

Multiplying both sides by $-\Delta K^{n+1}$ and integrating, we obtain

$$
\begin{aligned}
& \frac{\left\|\nabla K^{n+1}\right\|_{2}^{2}-\left\|\nabla K^{n}\right\|_{2}^{2}}{2 \tau}+\frac{\left\|\nabla\left(K^{n+1}-K^{n}\right)\right\|_{2}^{2}}{2 \tau} \\
\leq- & \left\|\Delta \nabla K^{n+1}\right\|_{2}^{2}+\theta_{c}\left\|\Delta K^{n+1}\right\|_{2}^{2} \\
& \quad+\left\langle\frac{\tilde{f}\left(u^{n+1}\right)-\tilde{f}\left(u^{n}\right)}{\tau},\left(-\Delta K^{n+1}\right)\right\rangle+\left\langle H_{1},\left(-\Delta K^{n+1}\right)\right\rangle,
\end{aligned}
$$

where

$$
H_{1}=\Delta^{2}\left(\tilde{f}\left(u^{n+1}\right)-\tilde{f}\left(u^{n}\right)\right)+\theta_{c} \Delta\left(\tilde{f}\left(u^{n+1}\right)-\tilde{f}\left(u^{n}\right)\right) .
$$

We first deal with the term $\left\langle\frac{\tilde{f}\left(u^{n+1}\right)-\tilde{f}\left(u^{n}\right)}{\tau},\left(-\Delta K^{n+1}\right)\right\rangle$. Rewrite

$$
\frac{\tilde{f}\left(u^{n+1}\right)-\tilde{f}\left(u^{n}\right)}{\tau}=\alpha_{n+1} \frac{u^{n+1}-u^{n}}{\tau}=\alpha_{n+1} \Delta K^{n}+\alpha_{n+1} \Delta\left(\tilde{f}\left(u^{n}\right)-\tilde{f}\left(u^{n+1}\right)\right),
$$


where

$$
\alpha_{n+1}=\int_{0}^{1} \tilde{f}^{\prime}\left(u^{n}+\theta\left(u^{n+1}-u^{n}\right)\right) d \theta
$$

Note that $\alpha_{n+1} \geq 0$. We then have

$$
\begin{aligned}
& \left\langle\frac{\tilde{f}\left(u^{n}\right)-\tilde{f}\left(u^{n-1}\right)}{\tau},\left(-\Delta K^{n+1}\right)\right\rangle \\
\leq & \left\langle\alpha_{n+1} \nabla\left(\tilde{f}\left(u^{n}\right)-\tilde{f}\left(u^{n+1}\right)\right), \Delta \nabla K^{n+1}\right\rangle+\left\langle\nabla \alpha_{n+1} \cdot \nabla\left(\tilde{f}\left(u^{n}\right)-\tilde{f}\left(u^{n+1}\right)\right), \Delta K^{n+1}\right\rangle \\
\leq & \frac{1}{8}\left\|\Delta \nabla K^{n+1}\right\|_{2}^{2}+2 \| \alpha_{n+1} \nabla\left(\tilde{f}\left(u^{n+1}-\tilde{f}\left(u^{n}\right)\right) \|_{2}^{2}\right. \\
& \quad+C_{\epsilon} \| \nabla \alpha_{n+1} \cdot \nabla\left(\tilde{f}\left(u^{n+1}-\tilde{f}\left(u^{n}\right)\right)\left\|_{\frac{4}{3}}^{2}+\epsilon\right\| \Delta K^{n+1} \|_{4}^{2} .\right.
\end{aligned}
$$

By Sobolev embedding, we have

$$
\left\|\Delta K^{n+1}\right\|_{4} \leq \mathrm{const}\left\|\nabla \Delta K^{n+1}\right\|_{2} .
$$

Also observe that

$$
\left\|\nabla \alpha_{n+1}\right\|_{4} \leq C_{A_{1}}
$$

Now taking $\epsilon>0$ sufficiently small, we obtain

$$
\begin{aligned}
& \left\langle\frac{\tilde{f}\left(u^{n+1}\right)-\tilde{f}\left(u^{n}\right)}{\tau},\left(-\Delta K^{n+1}\right)\right\rangle \\
\leq & \frac{1}{4}\left\|\Delta \nabla K^{n+1}\right\|_{2}^{2}+C_{A_{1}}\left\|\nabla\left(\tilde{f}\left(u^{n+1}\right)-\tilde{f}\left(u^{n}\right)\right)\right\|_{2}^{2} .
\end{aligned}
$$

By Lemma 2.1, we then have

$$
\left\langle\frac{\tilde{f}\left(u^{n+1}\right)-\tilde{f}\left(u^{n}\right)}{\tau},\left(-\Delta K^{n+1}\right)\right\rangle \leq \frac{1}{4}\left\|\Delta \nabla K^{n+1}\right\|_{2}^{2}+C_{A_{1}}\left\|\nabla\left(u^{n+1}-u^{n}\right)\right\|_{2}^{2} .
$$

Lemma 2.2. Recall

$$
H_{1}=\Delta^{2}\left(\tilde{f}\left(u^{n+1}\right)-\tilde{f}\left(u^{n}\right)\right)+\theta_{c} \Delta\left(\tilde{f}\left(u^{n+1}\right)-\tilde{f}\left(u^{n}\right)\right) .
$$

Assume

$$
\left\|u^{n}\right\|_{H^{5}} \leq C_{1},
$$

where $C_{1}>0$ is a constant. Then we have

$$
\begin{aligned}
& \left|\left\langle H_{1},\left(-\Delta K^{n+1}\right)\right\rangle\right| \\
\leq & \sqrt{\tau} \cdot C_{2}\left\|\Delta \nabla K^{n+1}\right\|_{2}+\theta_{c}\left\|\nabla\left(\tilde{f}\left(u^{n+1}\right)-\tilde{f}\left(u^{n}\right)\right)\right\|_{2}\left\|\Delta \nabla K^{n+1}\right\|_{2},
\end{aligned}
$$

where $C_{2}>0$ depends on $C_{1}$. 
Proof. We focus on the contribution of the term $\Delta^{2}\left(\tilde{f}\left(u^{n+1}\right)-\tilde{f}\left(u^{n}\right)\right)$. Since by assumption $\left\|u^{n}\right\|_{H^{5}} \leq C_{1}$, it is not difficult to obtain $\left\|u^{n+1}\right\|_{H^{5}} \leq \tilde{C}_{1}$ for some constant $\tilde{C}_{1}$ depending on $C_{1}$. We then write $\tilde{f}\left(u^{n+1}\right)-\tilde{f}\left(u^{n}\right)=\alpha_{n+1} \cdot\left(u^{n+1}-u^{n}\right)$ as before, and observe that

$$
u^{n+1}-u^{n}=-\frac{\tau \Delta^{2}+\tau \theta_{c} \Delta}{1+\tau \Delta^{2}+\tau \theta_{c} \Delta} u^{n}+\frac{\tau \Delta}{1+\tau \Delta^{2}+\tau \theta_{c} \Delta}\left(\tilde{f}\left(u^{n}\right)\right) .
$$

Clearly,

$$
\left\|u^{n+1}-u^{n}\right\|_{H^{3}} \leq \sqrt{\tau} \cdot \tilde{C}_{2},
$$

where $\tilde{C}_{2}$ depends on $C_{1}$. It is also not difficult to check that $\left\|\alpha_{n+1}\right\|_{H^{3}} \leq \tilde{C}_{3}$ for some $\tilde{C}_{3}$ depending on $C_{1}$. We then obtain

$$
\left|\left\langle\Delta^{2}\left(\tilde{f}\left(u^{n+1}\right)-\tilde{f}\left(u^{n}\right)\right), \Delta K^{n+1}\right\rangle\right| \leq \sqrt{\tau} C_{2}\left\|\Delta \nabla K^{n+1}\right\|_{2} .
$$

The desired estimate (2.16) then easily follows.

Now note

$$
\theta_{c}\left\|\Delta K^{n+1}\right\|_{2}^{2} \leq \frac{1}{4}\left\|\Delta \nabla K^{n+1}\right\|_{2}^{2}+C\left\|\nabla K^{n+1}\right\|_{2}^{2}
$$

Collecting all the estimates, we have

$$
\begin{aligned}
& \frac{\left\|\nabla K^{n+1}\right\|_{2}^{2}-\left\|\nabla K^{n}\right\|_{2}^{2}}{2 \tau} \\
& \leq-\frac{1}{4}\left\|\Delta \nabla K^{n+1}\right\|_{2}^{2}+\sqrt{\tau} \cdot C_{2}\left\|\Delta \nabla K^{n+1}\right\|_{2}+C\left\|\nabla K^{n+1}\right\|_{2}^{2}+C_{A_{1}}\left\|\nabla\left(u^{n+1}-u^{n}\right)\right\|_{2}^{2} .
\end{aligned}
$$

Now take $\tau$ sufficiently small such that $\sqrt{\tau} C_{2} \leq \frac{1}{4}, \tau C_{A_{1}} \leq \frac{1}{4}$. We discuss two cases.

Case 1: $\left\|\Delta \nabla K^{n+1}\right\|_{2} \leq 10$. In this case we call such $n$ a good point. In this case, no work is needed since by Poincaré inequality we have $\left\|\nabla K^{n+1}\right\|_{2} \leq 10$.

Case 2: $\left\|\Delta \nabla K^{n+1}\right\|_{2}>10$. In this case note that $\left\|\Delta \nabla K^{n+1}\right\|_{2}^{2} \geq 10\left\|\Delta \nabla K^{n+1}\right\|$. Thus we obtain

$$
\begin{aligned}
& \frac{\left\|\nabla K^{n+1}\right\|_{2}^{2}-\left\|\nabla K^{n}\right\|_{2}^{2}}{2 \tau} \\
& \leq-\frac{1}{400}\left\|\Delta \nabla K^{n+1}\right\|_{2}^{2}+C\left\|\nabla K^{n+1}\right\|_{2}^{2}+\frac{1}{4 \tau}\left\|\nabla\left(u^{n+1}-u^{n}\right)\right\|_{2}^{2} .
\end{aligned}
$$

Recall that we have shown (see (2.7) and (2.12))

$$
\tau \sum_{j=0}^{n}\left\|\nabla K^{j+1}\right\|_{2}^{2} \leq C, \quad \sum_{j=0}^{n}\left\|\nabla\left(u^{j+1}-u^{j}\right)\right\|_{2}^{2} \leq C .
$$


Now using (2.18) and summing backwards in $n$ until one meets a good $n$ or $n=0$, we then obtain

$$
\sup _{0 \leq j \leq n}\left\|\nabla K^{j+1}\right\|_{2} \leq C<\infty
$$

\subsection{Control of $\left\|g^{n+1}-\overline{g^{n+1}}\right\|_{2}$}

We shall use $\left\|\nabla K^{n+1}\right\|_{2} \leq C$, which gives $\left\|K^{n+1}-\overline{K^{n+1}}\right\|_{2} \leq C$. Write

$$
K^{n+1}-\overline{K^{n+1}}=-\Delta u^{n+1}-\theta_{c}\left(u^{n+1}-\overline{u^{n+1}}\right)+\theta\left(g^{n+1}-\overline{g^{n+1}}\right) .
$$

Multiplying both sides by $g^{n+1}-\overline{g^{n+1}}$, integrating (note the simple inequality $\left.\left|\left\langle u^{n+1}, g^{n+1}-\overline{g^{n+1}}\right\rangle\right| \leq\left\|g^{n+1}-\overline{g^{n+1}}\right\|_{2}\right)$ and using the Cauchy-Schwartz inequality, we obtain

$$
\left\|g^{n+1}-\overline{g^{n+1}}\right\|_{2} \leq C
$$

In the above derivation we used the fact that

$$
\int_{\mathbb{T}^{2}}\left(-\Delta u^{n+1}\right)\left(g^{n+1}-\overline{g^{n+1}}\right) d x=\int_{\mathbb{T}^{2}}\left|\nabla u^{n+1}\right|^{2} \frac{\theta}{1-\left(u^{n+1}\right)^{2}} d x \geq 0 .
$$

\subsection{Control of $\overline{g^{n+1}}$ and $\overline{K^{n+1}}$}

Lemma 2.3. Assume $\|g-\bar{g}\|_{2} \lesssim 1$. Let $u=\tanh (g)$ and $|\bar{u}|<1$. Then

$$
|\bar{g}| \lesssim(1-|\bar{u}|)^{-\frac{1}{2}}
$$

Proof. If $|\bar{g}| \leq 10$ we are done. Now we assume $\bar{g}=M \geq 10$. Since $\|g-\bar{g}\|_{2} \lesssim 1$, we obtain

$$
\operatorname{Leb}\{x \in \Omega: g(x) \leq M / 2\} \lesssim M^{-2}
$$

Now

$$
\begin{aligned}
\bar{u} \operatorname{Leb}(\Omega) & =\int_{g(x) \geq \frac{M}{2}} d x+\int_{g(x) \geq \frac{M}{2}}(u(x)-1) d x+\int_{g(x)<\frac{M}{2}} u(x) d x \\
& =\operatorname{Leb}(\Omega)+\int_{g(x) \geq \frac{M}{2}}(u(x)-1) d x+\int_{g(x)<\frac{M}{2}}(u(x)-1) d x \\
& =\operatorname{Leb}(\Omega)+\mathcal{O}\left(e^{-\frac{M}{4}}\right)+\mathcal{O}\left(\frac{1}{M^{2}}\right) .
\end{aligned}
$$


Clearly then

$$
(1-\bar{u}) \operatorname{Leb}(\Omega) \lesssim M^{-2}
$$

Thus the desired inequality follows. Note that if $\bar{g} \leq-10$ we need to work with $-u$ and hence the bound of $\bar{g}$ depends on $(1-|\bar{u}|)^{-\frac{1}{2}}$.

Since $\overline{u^{n+1}}=\overline{u^{0}}$ is preserved in time and $\left|\overline{u^{0}}\right|<1$, Lemma 2.3 implies that $\left|\overline{g^{n+1}}\right| \lesssim 1$. For the control of $\overline{K^{n+1}}$, recall that

$$
K^{n+1}=-\Delta u^{n+1}-\theta_{c} u^{n+1}+\theta g^{n+1} .
$$

Clearly then

$$
\left|\overline{K^{n+1}}\right| \leq \theta_{c}\left|\overline{u^{n+1}}\right|+\theta\left|\overline{g^{n+1}}\right| \lesssim 1
$$

2.6 Control of $\|u\|_{H^{3}},\|g\|_{H^{3}},\|\tilde{f}(u)\|_{H^{3}},\left\|\frac{1}{1-u^{2}}\right\|_{\infty},\left\|\partial\left(\frac{1}{1-u^{2}}\right)\right\|_{\infty}$ and $\left\|\partial^{2}\left(\frac{1}{1-u^{2}}\right)\right\|_{\infty-}$

Here $g=g^{j}$ and $u=u^{j}, 1 \leq j \leq n+1$. The obtained estimates will be uniform in $j$. See the subsection "Notation" for the definition of $\|f\|_{\infty_{-}}$.

We shall explain the argument for $j=n+1$. It is clear from the argument below that the estimates will be uniform in $j$.

Since $\left\|\nabla K^{n+1}\right\|_{2} \lesssim 1$ and we have the control of $\overline{K^{n+1}}$, it follows that

$$
\left\|K^{n+1}\right\|_{p} \lesssim \sqrt{p}, \quad \forall 2 \leq p<\infty .
$$

By using (2.26) (multiply both sides by $\left|g^{n+1}\right|^{p-2} g^{n+1}$ and integrate by parts), we then get

$$
\left\|g^{n+1}\right\|_{p} \lesssim \sqrt{p}
$$

This implies for any $C>0$,

$$
\left\|e^{C\left|g^{n+1}\right|}\right\|_{\infty-} \lesssim 1
$$

Since

$$
\frac{1}{1-\left(u^{n+1}\right)^{2}} \lesssim e^{2\left|g^{n+1}\right|}
$$

we also get

$$
\left\|\frac{1}{1-\left(u^{n+1}\right)^{2}}\right\|_{\infty-} \lesssim 1
$$


By using (2.26), we also get $\left\|\Delta u^{n+1}\right\|_{\infty_{-}} \lesssim 1$. This easily implies

$$
\left\|\partial^{2}\left(\frac{1}{1-\left(u^{n+1}\right)^{2}}\right)\right\|_{\infty-} \lesssim 1
$$

By Sobolev embedding, we obtain $\left\|\frac{1}{1-\left(u^{n+1}\right)^{2}}\right\|_{\infty} \lesssim 1$. Since

$$
\left|\ln \left(\frac{1+x}{1-x}\right)\right| \lesssim \frac{1}{1-x^{2}} \quad \text { for } \quad|x|<1
$$

we also obtain $\left\|g^{n+1}\right\|_{\infty} \lesssim 1$. Since

$$
\left\|\nabla g^{n+1}\right\|_{2} \lesssim\left\|\frac{1}{1-\left(u^{n+1}\right)^{2}} \nabla u^{n+1}\right\|_{2} \lesssim 1
$$

by using (2.26), we obtain $\left\|u^{n+1}\right\|_{H^{3}} \lesssim 1$. This further implies that

$$
\left\|\nabla g^{n+1}\right\|_{H^{2}} \lesssim\left\|\frac{1}{1-\left(u^{n+1}\right)^{2}} \nabla u^{n+1}\right\|_{H^{2}} \lesssim 1 \text {. }
$$

It is also clear that $\left\|\tilde{f}\left(u^{n+1}\right)\right\|_{H^{3}} \lesssim 1$.

\subsection{Control of $\left\|u^{n+1}\right\|_{H^{5}}$}

Here we shall exploit the discrete smoothing effect. Denote

$$
\begin{aligned}
u^{n+1} & =\frac{1}{1+\tau \Delta^{2}+\tau \theta_{c} \Delta} u^{n}+\frac{\tau \Delta}{1+\tau \Delta^{2}+\tau \theta_{c} \Delta}\left(\tilde{f}\left(u^{n}\right)\right) \\
& =: T_{0} u^{n}+T_{1} f^{n} .
\end{aligned}
$$

Iterating the above gives

$$
u^{n+1}=T_{0}^{J+1} u^{n-J}+\sum_{j=1}^{J} T_{0}^{j} T_{1} f^{n-j}+T_{1} f^{n} .
$$

In the estimate below, we shall use the uniform estimate:

$$
\left\|u^{0}\right\|_{H^{5}}+\sup _{0 \leq j \leq n}\left(\left\|u^{j}\right\|_{H^{3}}+\left\|f^{j}\right\|_{H^{3}}\right) \leq B_{1}<\infty .
$$




\subsubsection{Discrete smoothing estimates}

We first prove two auxiliary lemmas needed for the higher order estimates later. In a slightly more general setup, we assume for some $s \geq 0$,

$$
\sup _{0 \leq j \leq n}\left\|\widehat{h^{j}}(k)|k|^{s}\right\|_{l_{k}^{\infty} \leq \alpha_{1}<\infty}
$$

Define

$$
v=\sum_{j=1}^{n} T_{0}^{j} T_{1} h^{j}
$$

Lemma 2.4. We have

$$
\left\|\widehat{v}(k)|k|^{s+2}\right\|_{l_{k}^{\infty}} \leq \alpha_{1} .
$$

Consequently

$$
\|v\|_{H^{s+0.9}\left(\mathbb{T}^{2}\right)} \leq \alpha_{2}<\infty,
$$

where $\alpha_{2}>0$ depends only on $\alpha_{1}$.

Proof. Observe that

$$
\left|\widehat{T}_{0}(k) \widehat{T_{1}^{j}}(k)\right| \leq\left(\frac{1}{1+\tau|k|^{4}}\right)^{j} \cdot \frac{\tau|k|^{2}}{1+\tau|k|^{4}}
$$

Then for each $k \neq 0$, we have

$$
|\widehat{v}(k)| \leq \alpha_{1}|k|^{-s} \sum_{j=1}^{n}\left(\frac{1}{1+\tau|k|^{4}}\right)^{j} \cdot \frac{\tau|k|^{2}}{1+\tau|k|^{4}} \leq \alpha_{1}|k|^{-s-2} .
$$

Thus $\|v\|_{H^{s+0.9}\left(\mathbb{T}^{2}\right)}$ is bounded.

Lemma 2.5. Assume $0<\tau \leq 1$ and $4 \leq J \tau<5$. Then

$$
\left\|T_{0}^{J} g\right\|_{H^{16}\left(\mathbb{T}^{2}\right)} \lesssim\|g\|_{2} .
$$

Proof. Observe that $J \geq 4$ and

$$
\left|\widehat{T_{0}^{J}}(k)\right| \leq\left(1+|k|^{4} \frac{J \tau}{J}\right)^{-J} \leq\left(1+|k|^{4} \frac{4}{J}\right)^{-J} \leq\left(1+|k|^{4}\right)^{-4}
$$

Here we used the simple inequality that $h(x)=\left(1+\frac{1}{x}\right)^{x}$ is monotonically increasing in $x>0$. Clearly the desired inequality then follows. 


\subsubsection{Higher order estimates}

Now we discuss two cases.

Case 1: $n \tau \leq 20$. In this case we take $J=n$. By Lemma 2.4, we have

$$
\left\||k|^{5} \widehat{u^{n+1}}(k)\right\|_{l_{k}^{\infty}} \leq C .
$$

By a similar estimate we also obtain

$$
\sup _{0 \leq j \leq n+1}\left\||k|^{5} \widehat{u^{j}}(k)\right\|_{l_{k}^{\infty}} \leq C .
$$

It follows that

$$
\sup _{0 \leq j \leq n+1}\left\||k|^{5} \widetilde{\left(u^{j}\right)}(k)\right\|_{l_{k}^{\infty}} \leq C .
$$

By using Lemma 2.4 again, we obtain

$$
\left\|u^{n+1}\right\|_{H^{5}} \leq C .
$$

Case 2: $n \tau>20$. Since $0<\tau \leq 1$, we can choose an integer $J \geq 4$ such that $4 \leq J \tau<5$. By using Lemma 2.5, we have

$$
\left\|T_{0}^{J+1} u^{n-J}\right\|_{H^{10}} \leq C .
$$

The inhomogeneous term containing $f^{n-j}$ can be handled in the same way as in Case 1. Thus in this case we obtain $\left\|u^{n+1}\right\|_{H^{5}} \leq C$. This then completes the whole induction step. Theorem 1.1 is now proved.

\section{Proof of Corollary 1.1}

In this section we give the proof of Corollary 1.1. Consider first $s=5$. For each small $\tau>0$ we denote $u^{n, \tau}=u^{n}$ as the numerical solution obtained with the help of Theorem 1.1. Define $v^{(\tau)} \in C_{t}^{0} H_{x}^{5}$ such that

$$
v^{(\tau)}(t):=\left\{\begin{array}{lll}
u^{n, \tau}, & \text { if } t=n \tau, & n \geq 0 \\
\left(n+1-\frac{t}{\tau}\right) u^{n, \tau}+\left(\frac{t}{\tau}-n\right) u^{n+1, \tau}, & \text { if } n \tau \leq t<(n+1) \tau, & n \geq 0 .
\end{array}\right.
$$

In yet other words, $v^{(\tau)}$ is the piece-wise linear interpolation of $\left(u^{n, \tau}\right)_{n \geq 0}$. Observe that for each $t \in(n \tau,(n+1) \tau)$, we have

$$
\partial_{t} v^{(\tau)}=\frac{u^{n+1, \tau}-u^{n, \tau}}{\tau}=-\nu \Delta^{2} u^{n+1, \tau}-\theta_{c} \Delta u^{n+1, \tau}+\Delta\left(\tilde{f}\left(u^{n, \tau}\right)\right) .
$$


By Theorem 1.1, we have

$$
\sup _{0<\tau \leq \tau_{0}}\left(\left\|v^{(\tau)}\right\|_{C_{t}^{0} H_{x}^{5}}+\left\|\partial_{t} v^{(\tau)}\right\|_{L_{t}^{\infty} H_{x}^{1}}\right) \lesssim 1
$$

In the above, the norms are evaluated on the space-time slab $[0, \infty) \times \mathbb{T}^{2}$, and we have used the fact that the quotients $\frac{u^{n+1, \tau}-u^{n, \tau}}{\tau}$ are uniformly bounded in $L_{t}^{\infty} H_{x}^{1}$.

To proceed further, we need the following variant of the usual Aubin-Lions compactness lemma.

Lemma 3.1. Suppose $\left(v_{n}\right)_{n \geq 1}$ is a sequence of functions defined on $(t, x) \in[0, \infty) \times \mathbb{T}^{2}$ such that $v_{n} \in C_{t}^{0} H_{x}^{5}, \partial_{t} v_{n} \in L_{t}^{\infty} H_{x}^{1}$ and

$$
\sup _{n \geq 1}\left(\left\|v_{n}\right\|_{C_{t}^{0} H_{x}^{5}}+\left\|\partial_{t} v_{n}\right\|_{L_{t}^{\infty} H_{x}^{1}}\right) \lesssim 1
$$

Then there exists $v_{*} \in C_{t}^{0} H_{x}^{5}$ with $\partial_{t} v_{*} \in L_{t}^{\infty} H_{x}^{1}$, and a subsequence $n_{j}$, such that for any given $T>0$, and any $1 \leq s^{\prime}<5$,

$$
\left\|v_{n_{j}}-v_{*}\right\|_{C_{t}^{0} H_{x}^{s^{\prime}}\left([0, T] \times \mathbb{T}^{2}\right)} \rightarrow 0 \quad \text { as } j \rightarrow \infty .
$$

Proof. Without loss of generality, we assume $T=1$. From the argument below together with a further diagonal argument, one can easily cover the general case $T>0$.

First, fix any $k \in \mathbb{Z}^{2}$ and consider $\widehat{v_{n}}(t, k)$ on the time interval $[0,1]$. By using Arzelà-Ascoli and using a diagonal argument, one can extract a subsequence, which we denote as $\widehat{v_{n_{j}}}(t, k)$, such that it converges to a continuous (in $t$ ) function $\widehat{v_{*}}(t, k)$ on $[0,1]$ for any fixed $k$. Furthermore, since $\left|\widehat{v_{n}}(t, k)\right| \lesssim(1+|k|)^{-5}$, one can obtain

$$
\left\|\widehat{v_{n_{j}}}(t, k)-\widehat{v_{*}}(t, k)\right\|_{L_{t}^{\infty} l_{k}^{2}} \rightarrow 0 \quad \text { as } j \rightarrow \infty .
$$

By using interpolation one can obtain the strong convergence in $C_{t}^{0} H^{s^{\prime}}$ for any $s^{\prime}<5$.

By using Lemma 3.1, we obtain that along some sequence $\tau_{j} \rightarrow 0$, there exits $u \in C_{t}^{0} H^{5}$ with $\partial_{t} u \in L_{t}^{\infty} H^{4}$, such that as $j \rightarrow \infty$,

$$
\left\|v^{\left(\tau_{j}\right)}-u\right\|_{C_{t}^{0} H^{4.5}\left([0, T] \times \mathbb{T}^{2}\right)} \rightarrow 0, \quad \forall T>0 .
$$

Now by (3.2), it is not difficult to check that for any test function $\psi \in C_{c}^{\infty}\left((0, T) \times \mathbb{T}^{2}\right)$, we have

$$
\begin{aligned}
& \quad \mid \int_{(0, T) \times \mathbb{T}^{2}} \psi(t, x)\left(\partial_{t} v^{(\tau)}+\nu \Delta^{2} v^{(\tau)}+\theta \Delta v^{(\tau)}-\Delta\left(\tilde{f}\left(v^{(\tau)}\right)\right) d x d t \mid\right. \\
& \leq \mathcal{O}(\tau) \rightarrow 0 \text { as } \tau \rightarrow 0 .
\end{aligned}
$$


This together with the regularity of $u$ implies that $u$ is the desired solution. Note that the strict phase separation and uniform Sobolev regularity of $u$ on the time interval $[0, \infty)$ follows by taking the limit. Thanks to strict phase separation, it is routine to check that our constructed solution is unique. We note that the general case $s>5$ can be obtained by a simple bootstrapping argument. We omit further details here and leave them to interested readers.

\section{Proof of Theorem 1.2}

In this section we carry out the error estimate in $L^{2}$.

\subsection{Auxiliary $L^{2}$ error estimate for near solutions}

Consider

$$
\begin{cases}\frac{v^{n+1}-v^{n}}{\tau}=-\nu \Delta^{2} v^{n+1}-\theta_{c} \Delta v^{n+1}+\Delta \tilde{f}\left(v^{n}\right)+\Delta \tilde{G}_{n}^{1}, & n \geq 0, \\ \frac{\tilde{v}^{n+1}-\tilde{v}^{n}}{\tau}=-\nu \Delta^{2} \tilde{v}^{n+1}-\theta_{c} \Delta \tilde{v}^{n+1}+\Delta \tilde{f}\left(\tilde{v}^{n}\right)+\Delta \tilde{G}_{2}^{n}, & n \geq 0, \\ v^{0}=v_{0}, \quad \tilde{v}^{0}=\tilde{v}_{0}, & \end{cases}
$$

where $v_{0}$ and $\tilde{v}_{0}$ have the same mean and $\tilde{f}(z)=\frac{\theta}{2} \ln \left(\frac{1+z}{1-z}\right)$. For simplicity we shall make a slightly stronger assumption

$$
0<\tau \leq \frac{\nu}{4 \theta_{c}^{2}},
$$

so that the operator $\left(1+\tau \nu \Delta^{2}+\theta_{c} \tau \Delta\right)$ is invertible and consequently $v^{n+1}, \tilde{v}^{n+1}$ are well-defined for all $n \geq 0$. Denote

$$
\tilde{G}^{n}=\tilde{G}_{1}^{n}-\tilde{G}_{2}^{n} .
$$

Proposition 4.1. Assume $0<\tau \leq \frac{\nu}{4 \theta_{c}^{2}}$. For solutions of (4.1), assume for some $\delta_{1} \in(0,1)$,

$$
\sup _{n \geq 0}\left\|v^{n}\right\|_{\infty} \leq 1-\delta_{1}, \quad \sup _{n \geq 0}\left\|\tilde{v}^{n}\right\|_{\infty} \leq 1-\delta_{1} .
$$

Then for any $m \geq 1$, we have

$$
\left\|v^{m}-\tilde{v}^{m}\right\|_{2}^{2} \leq \exp \left(m \tau \cdot \frac{C_{1}}{\nu}\right) \cdot\left(\left\|v_{0}-\tilde{v}_{0}\right\|_{2}^{2}+\frac{2 \tau}{\nu} \sum_{n=0}^{m-1}\left\|\tilde{G}^{n}\right\|_{2}^{2}\right),
$$

where $C_{1}>0$ is a constant depending only on $\left(\delta_{1}, \theta, \theta_{c}\right)$. 
Proof. Denote $e^{n}=v^{n}-\tilde{v}^{n}$. Then

$$
\frac{e^{n+1}-e^{n}}{\tau}=-\nu \Delta^{2} e^{n+1}-\theta_{c} \Delta e^{n+1}+\Delta\left(\tilde{f}\left(v^{n}\right)-\tilde{f}\left(\tilde{v}^{n}\right)\right)+\Delta \tilde{G}^{n} .
$$

Taking the $L^{2}$ inner product with $e^{n+1}$ on both sides, we get

$$
\begin{aligned}
& \frac{1}{2 \tau}\left(\left\|e^{n+1}\right\|_{2}^{2}-\left\|e^{n}\right\|_{2}^{2}+\left\|e^{n+1}-e^{n}\right\|_{2}^{2}\right)+\nu\left\|\Delta e^{n+1}\right\|_{2}^{2}+\theta_{c}\left\langle e^{n+1}, \Delta e^{n+1}\right\rangle \\
= & \left\langle\tilde{G}^{n}, \Delta e^{n+1}\right\rangle+\left\langle\tilde{f}\left(v^{n}\right)-\tilde{f}\left(\tilde{v}^{n}\right), \Delta e^{n+1}\right\rangle .
\end{aligned}
$$

By the Cauchy-Schwartz inequality, we have

$$
\begin{aligned}
& \left|\left(\tilde{G}^{n}, \Delta e^{n+1}\right)\right| \leq \frac{\left\|\tilde{G}^{n}\right\|_{2}^{2}}{\nu}+\frac{\nu}{4}\left\|\Delta e^{n+1}\right\|_{2}^{2}, \\
& \left|\theta_{c}\left\langle e^{n+1}, \Delta e^{n+1}\right\rangle\right| \leq \frac{\theta_{c}^{2}}{\nu}\left\|e^{n+1}\right\|_{2}^{2}+\frac{\nu}{4}\left\|\Delta e^{n+1}\right\|_{2}^{2} \\
& \leq \frac{2 \theta_{c}^{2}}{\nu}\left\|e^{n+1}-e^{n}\right\|_{2}^{2}+\frac{2 \theta_{c}^{2}}{\nu}\left\|e^{n}\right\|_{2}^{2}+\frac{\nu}{4}\left\|\Delta e^{n+1}\right\|_{2}^{2} .
\end{aligned}
$$

Since $\max \left\{\left\|v^{n}\right\|_{\infty},\left\|\tilde{v}^{n}\right\|_{\infty}\right\} \leq 1-\delta_{1}$, we have

$$
\begin{aligned}
& \left\|\tilde{f}\left(v^{n}\right)-\tilde{f}\left(\tilde{v}^{n}\right)\right\|_{2} \leq C \cdot\left\|e^{n}\right\|_{2}, \\
& \left|\left\langle\tilde{f}\left(v^{n}\right)-\tilde{f}\left(\tilde{v}^{n}\right), \Delta e^{n+1}\right\rangle\right| \leq \frac{\nu}{4}\left\|\Delta e^{n+1}\right\|_{2}^{2}+\frac{C^{2}}{\nu}\left\|e^{n}\right\|_{2}^{2},
\end{aligned}
$$

where $C>0$ depends only on $\delta_{1}$ and $\theta$.

Collecting the estimates, we get

$$
\begin{aligned}
& \frac{\left\|e^{n+1}\right\|_{2}^{2}-\left\|e^{n}\right\|_{2}^{2}}{2 \tau}+\left(\frac{1}{2 \tau}-\frac{2 \theta_{c}^{2}}{\nu}\right)\left\|e^{n+1}-e^{n}\right\|_{2}^{2} \\
\leq & \frac{1}{\nu}\left\|\tilde{G}^{n}\right\|_{2}^{2}+\frac{C^{2}+2 \theta_{c}^{2}}{\nu}\left\|e^{n}\right\|_{2}^{2} .
\end{aligned}
$$

Define

$$
y_{n}=\left\|e^{n}\right\|_{2}^{2}, \quad \tilde{\alpha}=\frac{2 C^{2}+4 \theta_{c}^{2}}{\nu}, \quad \tilde{\beta}_{n}=\frac{2}{\nu}\left\|\tilde{G}^{n}\right\|_{2}^{2} .
$$

Then obviously

$$
\frac{y_{n+1}-y_{n}}{\tau} \leq \tilde{\alpha} y_{n}+\tilde{\beta}_{n}
$$

The desired result follows from the standard Gronwall inequality. 
Next we state and prove two lemmas needed for the proof of Theorem 1.2.

Lemma 4.1 (Discretization of the PDE solution). Let $t_{n}=n \tau, n \geq 0$. Let $u$ be the exact PDE solution to (1.1). Denote

$$
\tilde{f}(z)=\frac{\theta}{2} \ln \left(\frac{1+z}{1-z}\right)
$$

We have

$$
\frac{u\left(t_{n+1}\right)-u\left(t_{n}\right)}{\tau}=-\nu \Delta^{2} u\left(t_{n+1}\right)-\theta_{c} \Delta u\left(t_{n+1}\right)+\Delta\left(\tilde{f}\left(u\left(t_{n}\right)\right)\right)+\Delta \tilde{G}_{1}^{n},
$$

where

$$
\begin{aligned}
\tilde{G}_{1}^{n}=\frac{\nu}{\tau} \int_{t_{n}}^{t_{n+1}} \partial_{t} \Delta u \cdot\left(t-t_{n}\right) d t+\frac{\theta_{c}}{\tau} \int_{t_{n}}^{t_{n+1}} \partial_{t} u \cdot\left(t-t_{n}\right) d t \\
+\frac{1}{\tau} \int_{t_{n}}^{t_{n+1}} \partial_{t}(\tilde{f}(u)) \cdot\left(t_{n+1}-t\right) d t
\end{aligned}
$$

Similarly for a slightly different discretization, we have

$$
\frac{u\left(t_{n+1}\right)-u\left(t_{n}\right)}{\tau}=-\nu \Delta^{2} u\left(t_{n+1}\right)+\Delta\left(f\left(u\left(t_{n}\right)\right)\right)+\Delta \tilde{G}_{2}^{n}
$$

where

$$
\tilde{G}_{2}^{n}=\frac{\nu}{\tau} \int_{t_{n}}^{t_{n+1}} \partial_{t} \Delta u \cdot\left(t-t_{n}\right) d t+\frac{1}{\tau} \int_{t_{n}}^{t_{n+1}} \partial_{t}(f(u)) \cdot\left(t_{n+1}-t\right) d t
$$

Proof. Integrating the PDE for $u$ on the time interval $\left[t_{n}, t_{n+1}\right]$, we obtain

$$
\frac{u\left(t_{n+1}\right)-u\left(t_{n}\right)}{\tau}=\int_{t_{n}}^{t_{n+1}}\left(-\nu \Delta^{2} u(t)+\Delta(f(u(t)))\right) d s .
$$

Note that for a one-variable function $h=h(t)$, we have the formula

$$
\begin{aligned}
& \frac{1}{\tau} \int_{t_{n}}^{t_{n+1}} h(t) d t=h\left(t_{n}\right)+\frac{1}{\tau} \int_{t_{n}}^{t_{n+1}} h^{\prime}(t) \cdot\left(t_{n+1}-t\right) d t, \\
& \frac{1}{\tau} \int_{t_{n}}^{t_{n+1}} h(t) d t=h\left(t_{n+1}\right)+\frac{1}{\tau} \int_{t_{n}}^{t_{n+1}} h^{\prime}(t) \cdot\left(t_{n}-t\right) d t .
\end{aligned}
$$


By using the above formula, we have

$$
\begin{aligned}
& \int_{t_{n}}^{t_{n+1}}\left(-\nu \Delta^{2} u(t)\right) d t=-\nu \Delta^{2} u\left(t_{n+1}\right)+\frac{1}{\tau} \int_{t_{n}}^{t_{n+1}} \nu \partial_{t} \Delta^{2} u(t) \cdot\left(t-t_{n}\right) d t, \\
& \int_{t_{n}}^{t_{n+1}}\left(-\theta_{c} \Delta u(t)\right) d t=-\theta_{c} \Delta u\left(t_{n+1}\right)+\frac{1}{\tau} \int_{t_{n}}^{t_{n+1}} \theta_{c} \partial_{t} \Delta u(t) \cdot\left(t-t_{n}\right) d t, \\
& \int_{t_{n}}^{t_{n+1}} \Delta(\tilde{f}(u(t))) d t=\Delta\left(\tilde{f}\left(u\left(t_{n}\right)\right)\right)+\frac{1}{\tau} \int_{t_{n}}^{t_{n+1}} \partial_{t} \Delta(\tilde{f}(u(t))) \cdot\left(t_{n+1}-t\right) d t .
\end{aligned}
$$

Thus

$$
\frac{u\left(t_{n+1}\right)-u\left(t_{n}\right)}{\tau}=-\nu \Delta^{2} u\left(t_{n+1}\right)-\theta_{c} \Delta u\left(t_{n+1}\right)+\Delta\left(\tilde{f}\left(u\left(t_{n}\right)\right)\right)+\Delta \tilde{G}^{n}
$$

where

$$
\begin{aligned}
\tilde{G}^{n}=\frac{\nu}{\tau} & \int_{t_{n}}^{t_{n+1}} \partial_{t} \Delta u \cdot\left(t-t_{n}\right) d t+\frac{\theta_{c}}{\tau} \int_{t_{n}}^{t_{n+1}} \partial_{t} u \cdot\left(t-t_{n}\right) d t \\
& +\frac{1}{\tau} \int_{t_{n}}^{t_{n+1}} \partial_{t}(\tilde{f}(u)) \cdot\left(t_{n+1}-t\right) d t .
\end{aligned}
$$

The derivation of (4.10) is similar. We omit details.

Lemma 4.2. Let u be the PDE solution constructed in Corollary 1.1. Then we have

$$
\int_{0}^{\infty}\left(\left\|\partial_{t} u\right\|_{2}^{2}+\left\|\partial_{t} \Delta u\right\|_{2}^{2}\right) d t \lesssim 1
$$

Proof. The $L^{2}$-in time integrability of $\partial_{t} u$ comes from the energy identity, i.e.,

$$
\frac{d}{d t} \mathcal{E}(u(t))=-\left\|\partial_{t} u\right\|_{L_{x}^{2}}^{2} \Longrightarrow \mathcal{E}(u(T))+\int_{0}^{T}\left\|\partial_{t} u\right\|_{2}^{2} d t=\mathcal{E}\left(u_{0}\right), \quad \forall T>0
$$

Sending $T \rightarrow \infty$ then yields

$$
\int_{0}^{\infty}\left\|\partial_{t} u\right\|_{2}^{2} d t<\infty
$$

Next to obtain $L^{2}$-in time integrability of $\partial_{t} \Delta u$, we recall

$$
\partial_{t} u=\Delta \mu=\Delta\left(-\nu \Delta u-\theta_{c} u+\tilde{f}(u)\right) .
$$

Clearly,

$$
\partial_{t} \mu=-\nu \Delta^{2} \mu-\theta_{c} \Delta \mu+\tilde{f}^{\prime}(u) \Delta \mu
$$


Thanks to strict phase separation, we have $\tilde{f}^{\prime}(u) \geq 0$. Multiplying both sides of (4.13) by $-\Delta \mu$ and integrating by parts, we obtain

$$
\frac{1}{2} \partial_{t}\left(\|\nabla \mu\|_{2}^{2}\right) \leq-\nu\|\Delta \nabla \mu\|_{2}^{2}+\theta_{c}\|\Delta \mu\|_{2}^{2}
$$

Integrating in time and using the fact that $\Delta \mu=\partial_{t} u \in L_{t}^{2} L_{x}^{2}\left((0, \infty) \times \mathbb{T}^{2}\right)$, we obtain

$$
\int_{0}^{\infty}\|\Delta \nabla \mu\|_{2}^{2} d t<\infty
$$

Multiplying both sides of (4.13) by $\Delta^{2} \mu$ and integrating by parts, we then obtain

$$
\int_{0}^{\infty}\left\|\Delta^{2} \mu\right\|_{2}^{2} d t<\infty
$$

Here it should be noted that in deriving the above, we used the finiteness of $\|\Delta \mu\|_{2}$ which is clearly bounded since $u \in H^{5}$ and has strict phase separation. Since $\partial_{t} \Delta u=$ $\Delta^{2} \mu$, we then obtain

$$
\int_{0}^{\infty}\left\|\partial_{t} \Delta u\right\|_{2}^{2} d t<\infty .
$$

Thus, we complete the proof.

Proof of Theorem 1.2. We need to consider

$$
\left\{\begin{array}{l}
\frac{u^{n+1}-u^{n}}{\tau}=-\nu \Delta^{2} u^{n+1}-\theta_{c} \Delta u^{n+1}+\Delta\left(\tilde{f}\left(u^{n}\right)\right) \\
\partial_{t} u=-\nu \Delta^{2} u+\Delta(f(u)) \\
\tilde{u}^{0}=u_{0}, \quad u(0)=u_{0}
\end{array}\right.
$$

We first rewrite the PDE solution $u$ in the discretized form. By Lemma 4.1, we have

$$
\frac{u\left(t_{n+1}\right)-u\left(t_{n}\right)}{\tau}=-\nu \Delta^{2} u\left(t_{n+1}\right)-\theta_{c} \Delta u\left(t_{n+1}\right)+\Delta\left(\tilde{f}\left(u\left(t_{n}\right)\right)\right)+\Delta \tilde{G}^{n},
$$

where

$$
\begin{aligned}
\tilde{G}^{n}=\frac{\nu}{\tau} & \int_{t_{n}}^{t_{n+1}} \partial_{t} \Delta u \cdot\left(t-t_{n}\right) d t+\frac{\theta_{c}}{\tau} \int_{t_{n}}^{t_{n+1}} \partial_{t} u \cdot\left(t-t_{n}\right) d t \\
& +\frac{1}{\tau} \int_{t_{n}}^{t_{n+1}} \partial_{t}(\tilde{f}(u)) \cdot\left(t_{n+1}-t\right) d t
\end{aligned}
$$


By using strict phase separation and uniform Sobolev regularity of $u$, we have

$$
\begin{aligned}
\left\|\tilde{G}^{n}\right\|_{2} & \leq \nu \int_{t_{n}}^{t_{n+1}}\left\|\partial_{t} \Delta u\right\|_{2} d t+\theta_{c} \int_{t_{n}}^{t_{n+1}}\left\|\partial_{t} u\right\|_{2} d t+\int_{t_{n}}^{t_{n+1}}\left\|\partial_{t} u\right\|_{2} d t \cdot \sup _{|z| \leq 1-\delta_{1}}\left|\tilde{f}^{\prime}(z)\right| \\
& \lesssim\left(\int_{t_{n}}^{t_{n+1}}\left(\left\|\partial_{t} \Delta u\right\|_{2}^{2}+\left\|\partial_{t} u\right\|_{2}^{2}\right) d t\right)^{\frac{1}{2}} \cdot \sqrt{\tau}
\end{aligned}
$$

By Lemma 4.2, we obtain

$$
\sum_{n=0}^{m-1}\left\|\tilde{G}^{n}\right\|_{2}^{2} \lesssim \tau \int_{0}^{t_{m}}\left(\left\|\partial_{t} \Delta u\right\|_{2}^{2}+\left\|\partial_{t} u\right\|_{2}^{2}\right) d t \lesssim \tau .
$$

Thus, by Proposition 4.1, we get

$$
\left\|u^{m}-u\left(t_{m}\right)\right\|_{2}^{2} \lesssim e^{C t_{m}} \tau^{2}
$$

Thus we obtain (1.20).

\section{Concluding remarks}

In this paper we studied the Cahn-Hilliard equation with singular logarithmic potentials on the two-dimensional periodic torus. We analyzed a first order in time, semi-implicit numerical discretization scheme which treats the linear fourth-order dissipation term implicitly and the nonlinear term explicitly. Prior state of the art literature are concerned with implicit or partially implicit methods for which phase separation and energy stability are established under nearly optimal conditions. For semi-implicit type methods, these issues were long standing open problems. In this work we developed a new theoretical framework and proved strict phase separation and energy stability for all time under mild constraints on the time step and initial data. We also carried out a rigorous error analysis which is done for the first time for semi-implicit methods on Cahn-Hilliard equations with singular potentials. It is expected our theoretical framework can be refined and generalized to cover many other similar problems. Research is now underway to investigate several directions including the stability and error analysis of higher-order methods, general thin-film type problems with singular potentials, various time-splitting methods, and adaptive time-stepping methods.

\section{Acknowledgements}

The first author's work was supported in part by Hong Kong RGC grant GRF Nos. 16307317 and 16309518. The second author's work is partially supported by 
the NSFC grants Nos. 11731006 and K20911001, NSFC/RGC No. 11961160718, and the Science Challenge Project (No. TZ2018001).

\section{References}

[1] H. Abels and M. Wilke, Convergence to equilibrium for the Cahn-Hilliard equation with a logarithmic free energy, Nonlinear Anal., 67 (2007), pp. 3176-3193.

[2] J. W. Cahn and J. E. Hilliard, Free energy of a nonuniform system. I. Interfacial free energy, J. Chem. Phys., 28 (1958), pp. 258-267.

[3] L. Cherfils, A. Miranville, and S. Zelik, The Cahn-Hilliard equation with logarithmic potentials, Milan J. Math., 79(2) (2011), pp. 561-596.

[4] M. Copetti and C. Elliott, Numerical analysis of the Cahn-Hilliard equation with a logarithmic free energy, Numer. Math., 63 (1992), pp. 39-65.

[5] W. Chen, C. Wang, X. Wang, and S. Wise, Positivity-preserving, energy stable numerical schemes for the Cahn-Hilliard equation with logarithmic potential, J. Comput. Phys., 3 (2019), 100031.

[6] A. Debussche and L. Dettori, On the Cahn-Hilliard equation with a logarithmic free energy, Nonlinear Anal., 24 (1995), pp. 1491-1514.

[7] C. M. Elliott and S. Luckhaus, A generalized diffusion equation for phase separation of a multi-component mixture with interfacial energy, SFB 256 preprint No. 195, University of Bonn, 1991.

[8] M. I. M. Copetti and C. M. Elliott, Numerical analysis of the Cahn-Hilliard equation with a logarithmic free energy, Numer. Math., 63 (1992), pp. 39-65.

[9] J. F. Blowey, M. I. M. Copetti, and C. M. Elliott, Numerical analysis of a model for phase separation of a multi-component alloy, IMA J. Numer. Anal., 16 (1996), pp. 111-139.

[10] G. Giacomin and J. L. Lebowitz, Phase segregation dynamics in particle systems with long range interaction I. Macroscopic limits, J. Statist. Phys., 87 (1997), pp. $37-61$.

[11] G. Giacomin and J. L. Lebowitz, Phase segregation dynamics in particle systems with long range interaction II. Interface motion, SIAM J. Appl. Math., 58 (1998), pp. $1707-1729$.

[12] P. W. Bates and J. Han, The Neumann boundary problem for a nonlocal CahnHilliard equation, J. Diffential Equations, 212 (2005), pp. 235-277.

[13] H. Gajewski and K. Zacharias, On a nonlocal phase separation model, J. Math. Anal. Appl., 286 (2003), pp. 11-31.

[14] N. Kenmochi, M. Niezgódka and I. Pawlow, Subdifferential operator approach to the Cahn-Hilliard equation with constraint, J. Diffential Equations, 117 (1995), pp. 320-356.

[15] A. Miranville and S. Zelik, Robust exponential attractors for Cahn-Hilliard type equations with singular potentials, Math. Methods Appl. Sci., 27 (2004), pp. 545- 
582.

[16] P. W. Bates, P. C. Fife, X. Ren, and X. Wang, Traveling waves in a convolution model for phase transitions. Arch. Rational Mech. Anal., 138(2) (1997), pp. 105-136.

[17] H. Wu and T. Fukao, Separation property and convergence to equilibrium for the equation and dynamic boundary condition of Cahn-Hilliard type with singular potential, ArXiv:1910.14177v1.

[18] G. Schimperna and H. Wu, On a class of sixth-order Cahn-Hilliard type equations with logarithmic potential, ArXiv:1909.01816v3.

[19] A. Miranville, The Cahn-Hilliard equation and some of its variants, AIMS Math., 2 (2017), pp. 479-544.

[20] T. Tang, Revisit of semi-implicit schemes for phase-field equations, Anal. Theory Appl., 36 (2020), pp. 235-242.

[21] X. Yang and G. Zhang, Numerical approximations of the Cahn-Hilliard and AllenCahn equations with general nonlinear potential using the invariant energy quadratization approach, ArXiv:1712.02760v1. 